\title{
A HIGH-POWER TARGET EXPERIMENT AT THE CERN PS
}

FERMILAB-CONF-07-593-APC

\author{
H.G Kirk*, H.-J. Park, T. Tsang, BNL, Upton, NY 11973, USA \\ A. Fabich, I. Efthymiopoulos, F. Haug, J. Lettry, M. Palm, \\ CERN, CH-1211 Genève 23, Switzerland \\ N. Mokhov, S. Striganov, FNAL, Batavia, IL, 60510, USA \\ A. Carroll, V.B. Graves, P.T. Spampinato, ORNL, Oak Ridge, TN 37831, USA \\ K.T. McDonald, Princeton University, Princeton, NJ 08544, USA \\ J.R.J. Bennett, O. Cannetta, P. Loveridge, CCLRC, RAL, Chilton, OX11 0QX, UK
}

\section{Abstract}

We test a target concept devised for the purpose of producing copious secondary pions and capturing the muon decay products. This experiment is designed to test the target system for a neutrino factory or muon collider and consists of a free flowing mercury stream embedded in a high-field solenoid. Key components are described.

\section{INTRODUCTION}

The U.S. Neutrino Factory and Muon Collider Collaboration (refereed to here after as the Muon Collaboration) has been pursuing a solution to generate an intense muon beam suitable for pursuing advanced physics experiments based on circulating muon beams in storage rings. In the case of a Muon Collider this goal is realized through colliding, counter-circulating muon beams. For a Neutrino Factory, intense neutrino beams can be generated through the decay of circulating muon beams in suitably placed long straight sections.

A key component of this program is establishing the technology to generate a copious source of muons. A proposed solution $[1,2]$ consists of converting a powerful primary proton beam into an intense pion beam. These pions are then conducted into a decay channel where the muon decay products can be collected. Primary proton beams with megawatt class power are being considered, in particular, for a Neutrino Factory, 4-MW proton beam power is the baseline option.

Designing a target system capable of handling such beam power is a challenging endeavor. Consequently, the Muon Collaboration has embarked on an R\&D program with the goal to develop and demonstrate the required technology.

\section{THE TARGET CONCEPT}

The basic concept for muon production consists of utilizing a high- $Z$ target which will generate copious low-energy pions of both signs and then capturing them within the confines of an high field solenoid. The proposed target material

*hkirk@bnl.gov
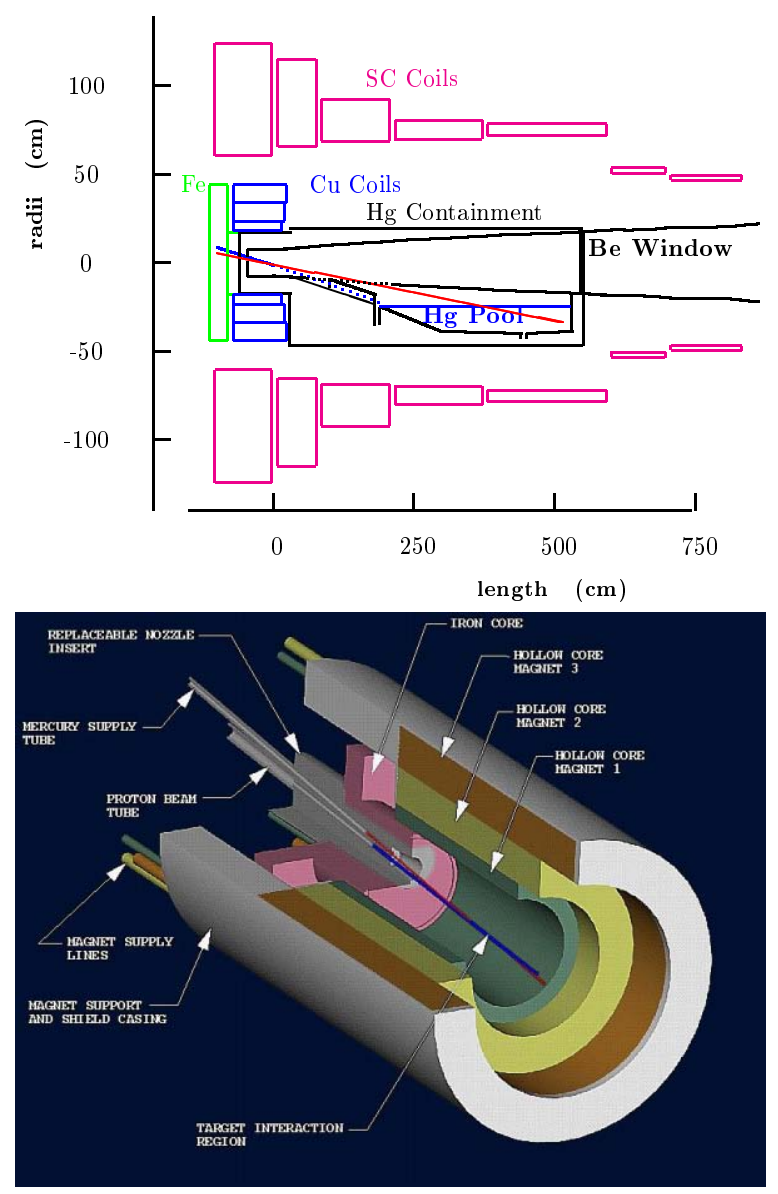

Figure 1: Concept of a continuous mercury jet target for an intense proton beam. The jet and beam are tilted by $100 \mathrm{mrad}$ and $67 \mathrm{mrad}$, respectively, with respect to a $20-\mathrm{T}$ solenoid magnet that conducts low-momentum pions into a decay channel.

is a liquid mercury jet which can easily be replenished after each interaction with the proton beam. Solenoid fields of 15-20 T will be adequate to capture most pions with $P_{\perp}$ up to $220 \mathrm{MeV} / c$. These pions are then conducted along a tapered solenoid channel in which the muon decay products of the pions are contained and further transported into phase space shaping systems such as phase rotation in lon- 
gitudinal phase space and cooling channels in which the transverse emittance of the muons is reduced. Figures 1a and $b$ show the schematics of the capture scheme. Figure 2 gives a view of the infrastructure required to support a target system of this type.

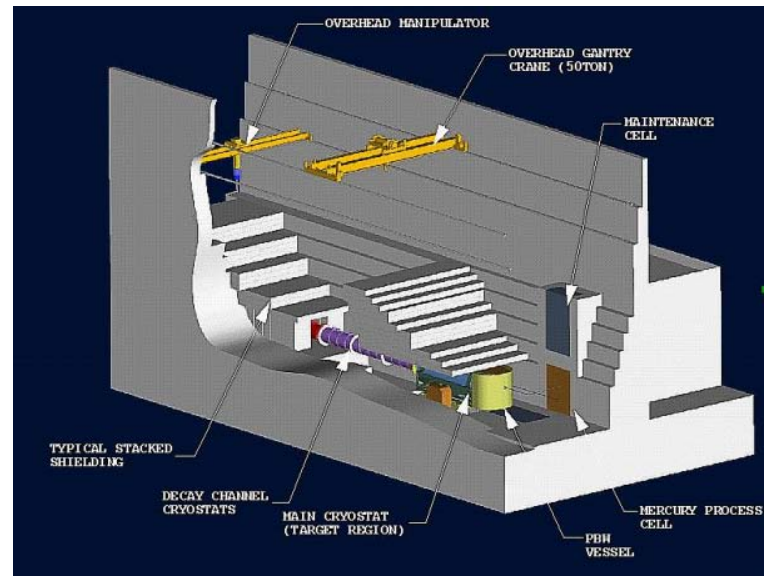

Figure 2: Concept of the target vault for a 4-MW mercury jet target [3].

There are key technical issues associated with this proposed targeting solution. The performance of the mercury jet will be effected by the presence of the magnetic field. The dispersal of the mercury after the interaction with the proton beam needs to be studied. Other issues include how much displaced in time can micro-bunches be and still have full pion production and the corollary question, how soon can the jet be reconstituted in order to accept a subsequent beam pulse. These and other issues represent the basis for the Muon Collaboration establishing its targetry R\&D program.

\section{THE TARGET EXPERIMENT}

Initial experiments were conducted at the BNL AGS [4] in which the characteristics of the interaction of an $\mathrm{Hg}$ jet with a $24-\mathrm{GeV}$ proton beam was first studied. These studies gave encouraging but limited results. First, the $\mathrm{Hg}$ jet velocity was only $2.5 \mathrm{~m} / \mathrm{s}$, whereas one of order $20 \mathrm{~m} / \mathrm{s}$ is required for high rep. rate $(\approx 50-\mathrm{Hz})$ operations. Also, the previous experiments did not include the presence of a magnetic field. With this in mind, a proposal [5] was submitted and subsequently accepted for testing at CERN using the $24-\mathrm{GeV}$ proton beam from the CERN PS. An initial discussion of the concept for this experiment has already been published [6].

Two key experimental components of this experiment, the 15-T Pulsed solenoid and the $\mathrm{Hg}$ injection system, have been described elsewhere [7, 8]. The experimental floor layout of these systems are shown in Fig. 3 while the relationship of the equipment relative to the proton beam is shown in Fig. 4.

The beam spill will be varied with single turn extraction used for the 24-GeV PS proton beam. Beam pulses will

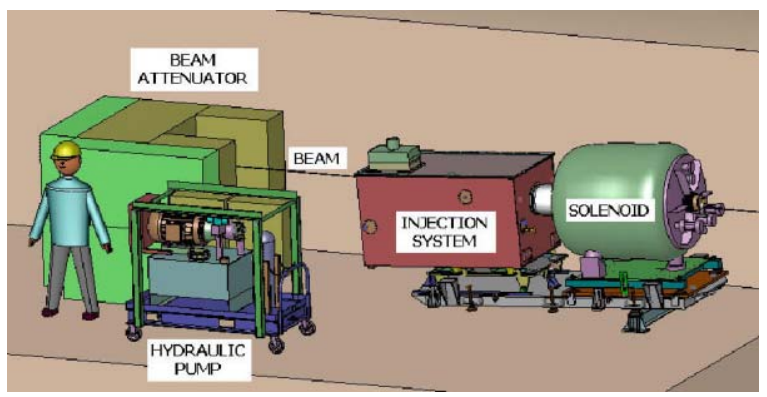

Figure 3: Layout of the high-power-target experiment.

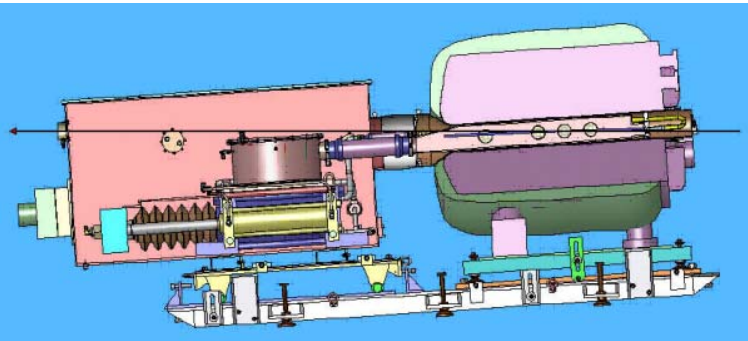

Figure 4: Cutaway view of the high-power-target experiment.

vary in intensity from 4 to $30 \times 10^{12}$ protons/pulse (4-30 TP). Multiple turn extractions can be realized provided that the primary proton beam energy is reduced to $14 \mathrm{GeV}$. The time interval between the extractions can be varied from 2 to $500 \mu \mathrm{s}$. We will exploit this machine capability by invoking a pump-probe technique to examine the particle production efficiency of the trailing (probe) pulse as a function of the time interval. This will give more detailed information concerning cavitation development in the mercury jet.

The particle production efficiencies of each micro-pulse will be examined through the use of various particle detectors arrayed about the interaction site. Two types of detectors will be used: diamond based PIN-diode and Aluminum Cathode Electron Multipliers (ACEM). We use the particle production code MARS as a guide to predict the expected particle fluences and time distributions at selected locations, as shown in Fig. 5.

\section{CONCLUSIONS}

The CERN target experiment is structured to provide a proof-of-principle verification of the Muon Collaboration target concept. Principle components have been installed and the running of the experiment is expected to begin this summer.

\section{ACKNOWLEDGMENTS}

This work was supported in part by the US DOE Contract NO. DE-AC02-98CH10886. 


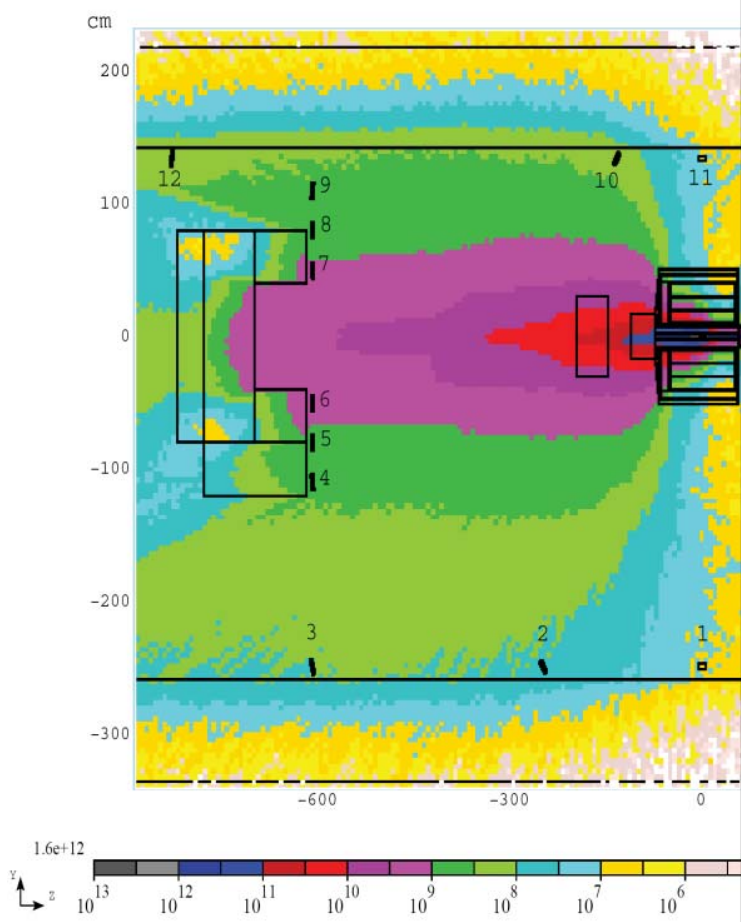

Figure 5: Secondary pion fluxes for a pulse of $3 \times 10^{13}$ 24-GeV protons on an $\mathrm{Hg}$ target, shown in the horizontal plane at beam height, according to the MARS code. The positions of 12 particle detectors are indicated in the figure.

\section{REFERENCES}

[1] H.G. Kirk, Targetry for a $\mu^{+} \mu^{-}$Collider, Proc. 1999 Part. Accel. Conf., p. 3030.

[2] K.T. McDonald et al., The Primary Target Facility for a Neutrino Factory Based on Muon Beams, Proc. 2001 Part. Accel. Conf., p. 1583.

[3] P. Spampinato, et al., Target Enclosure and System Design for a Mercury-Target Neutrino Producing Facility, Proc. 2005 Part. Accel. Conf., p. 1580.

[4] H.G. Kirk, et al., Target Studies with BNL E951 at the AGS, Proc. 2001 Part. Accel. Conf., p. 1535.

[5] J.R.J. Bennett, et al., Studies of a Target System for a 4-MW, $24 \mathrm{GeV}$ Proton Beam, Proposal to the CERN INTC Committee, INTC-P-186 (April 26, 2004),

http://puhep1.princeton.edu/mumu/target/cern_proposal.pdf

[6] H.G. Kirk, et al., A High-Power Target Experiment, Proc. 2005 Part. Accel. Conf., p. 3745.

[7] H.G. Kirk, et al., A High-field Pulsed Solenoid for Liquid Metal Target Studies, Proc. 2003 Part. Accel. Conf., p. 1631.

[8] V.B. Graves, et al., Systems Testing of a Free Hg Jet System for Use in a High-Power Target Experiment, these Proceedings. 